\title{
The Open University's first one-day symposium on treatment-emergent neuroendocrine prostate cancer
}

\author{
Rebecca L Mather ${ }^{1}$ (D) Henry Andrews ${ }^{2}$, Hardev Pandha ${ }^{3}$, Elena Jachetti ${ }^{4}$, Jake Micallef ${ }^{5}$, \\ Yuzhuo Wang ${ }^{6,7,8}$ \& Francesco Crea*,1 \\ ${ }^{1}$ School of Life, Health \& Chemical Sciences, The Open University, Walton Hall, Milton Keynes, Buckinghamshire, MK7 6AA, UK \\ ${ }^{2}$ Urology Department, Milton Keynes University Hospital, Standing Way, Eaglestone, Milton Keynes, MK6 5LD, UK \\ ${ }^{3}$ Department of Clinical \& Experimental Medicine, Faculty of Health \& Medical Science, Leggett Building, Daphne Jackson Road, \\ University of Surrey, Guildford GU2 7WG, UK \\ ${ }^{4}$ Molecular Immunology Unit, Department of Research, Fondazione IRCCS Istituto Nazionale Tumori, 20133 Milano, Italy \\ ${ }^{5}$ VolitionRx, 93-95 Gloucester Place, London, W1U 6JQ, UK \\ ${ }^{6}$ Experimental Therapeutics, BC Cancer Research Centre, Vancouver, BC, V5Z 4E6, Canada \\ ${ }^{7}$ The Vancouver Prostate Centre, Vancouver General Hospital, Vancouver, BC, V5Z 1M9, Canada \\ ${ }^{8}$ Department of Urologic Sciences, University of British Columbia, Vancouver, BC, V5Z 1M9, Canada \\ *Author for correspondence: francesco.crea@open.ac.uk
}

The Open University's first one-day symposium on treatment-emergent neuroendocrine prostate cancer attracted world-leading figures, early career researchers and industry colleagues. The symposium proved insightful into the 'real-world' impact and current problems faced in the diagnosis and treatment of neuroendocrine prostate cancer. It was important for this meeting to take place as the incidence of neuroendocrine prostate cancer is increasing due to the widespread use of next-generation androgen deprivation drugs. The symposium discussions proposed new molecularly driven deadlines to accelerate research and improved the treatment of this deadly and poorly recognized malignancy.

First draft submitted: 22 November 2019; Accepted for publication: 13 January 2020; Published online: 22 January 2020

Keywords: cancer biomarkers $\bullet$ epigenetics $\bullet$ long noncoding RNAs $\bullet$ neuroendocrine prostate cancer $\bullet$ osteopontin - tumor microenvironment

Treatment-emergent neuroendocrine prostate cancer (NEPC) is a recently identified clinicopathological subtype of prostatic neoplasm, which is characterized by dismal prognosis and complete resistance to any hormonal therapy [1]. The Open University hosted its first one-day symposium entitled 'Treatment-emergent neuroendocrine prostate cancer' on 25 June 2019. The symposium attracted 39 clinicians, researchers and students from across the UK and internationally, who gathered to share, hear and debate the very latest developments in neuroendocrine prostate cancer research.

The program featured keynotes from world-leading figures, early career researchers and industry colleagues proving impactful insights on current problems faced in the diagnosis and treatment of NEPC. A broad range of topics were covered including clinical diagnosis, molecular mechanisms of disease progression and the development of novel biomarkers and therapeutics.

\section{Highlights}

Highlights of the first session included an insight into the clinical progression of prostate cancer. Professor Hardev Pandha, Director of the Surrey Cancer Centre (Surrey, UK), described the clinical presentation of prostate cancer and also his experience managing neuroendocrine disease. Pandha highlighted the difficulties associated with diagnosing this complex pathology and also the problems associated with second-line anti-androgens (abiraterone, enzalutamide). The lecture concluded by recommending re-biopsy of patients with suspected NEPC in order to improve accurate diagnosis (i.e., not waiting for treatment failure) and in doing so treating patients more specifically. Pandha also stressed on increasing research material for understanding this disease better.

Future Medicine 
Following Pandha's presentation, Dr Francesco Crea, Senior Lecturer at The Open University (Milton Keynes, UK), discussed the cellular origins of prostate cancer and the 'classical' model of NEPC development [2]. Classically, NEPC was thought to arise from oncogenic transformation of basal cells, distinct from the oncogenic transformation of luminal cells which give rise to prostatic adenocarcinoma [3]. However, this model has been challenged by several studies $[4,5]$. These studies provide evidence that prostatic adenocarcinomas treated with second-line anti-androgens may give rise to either CRPC, or NEPC through a largely mechanistically unknown transdifferentiation process. Both Pandha and Crea stated that the incidence of NEPC is likely to rise with increased use of second-line anti-androgens and recommended re-biopsying patients with suspected NEPC after therapy.

To conclude the morning's clinical session, a discussion including Dr Jake Micallef from biotechnology company Volition (Isnes Belgium), Pandha, Crea and Mr Henry Andrews, a urologist from Milton Keynes Hospital then took place. The panel briefly introduced themselves, following which the audience had an insightful and open discussion with the panel on how the NEPC community can work together to change current guidelines. This change in guidelines is necessary to improve not only rapid diagnosis, but also the unnecessary use of ineffective treatments. It is only then that we can begin to identify effective treatments against this disease. To underscore the importance of new guidelines, Pandha presented two clinical cases and in both cases, biopsies were taken from CRPC patients that had shown progression after abiraterone or enzalutamide treatment. In both cases, the hormonal therapy was substituted by platinum-based chemotherapy. Pandha stated that since June 2018 his cancer center had identified and treated seven NEPC patients, who were identified by soft-tissue biopsies. For the Surrey NEPC project Pandha indicated that they would radiologically monitor 100 CRPC patients and biopsy when clinically indicated, as well as work to develop serum markers in order to improve diagnosis.

In the afternoon session, the molecular mechanisms of NEPC initiation and progression were discussed. Professor Yuzhuo Wang from the BC Cancer Agency (Vancouver, Canada) gave a lecture on his lab's approach to the challenge of targeting NEPC using a pipeline, ranging from discovery to drug development. Wang first described the expression and function of HP1a knock-down in various NEPC models demonstrating that this protein regulates NEPC cell survival and growth [6]. Wang further described that HP1a also induces the neuroendocrine phenotype in prostatic adenocarcinoma cells upon overexpression. Mechanistically, Wang then described that HP1a works by enriching histone 3 lysine trimethylation on AR and REST promoters which represses their expression, promoting the NEPC phenotype. Concluding this data, Wang suggested HP1a as a serum marker and as a druggable target to prevent transdifferentiation to NEPC and for NEPC. Wang then described another target identified by his lab after going back to one of the fundamental hallmarks of cancer: evasion of the immune response. MCT4, a transporter protein, was identified in prostate cancer as a possible immune regulator [7]. This protein is a transporter involved in glycolytic phenotypes. By designing anti-sense oligonucleotides against this protein, the study demonstrated enhanced anti-cancer immunity in PC-3 bearing nude mice and in a first-generation PDX model [8]. This model demonstrated increased tumor-associated NK cells after anti-sense treatments (PC-3) and in proliferation of patient tumor-associated $\mathrm{CD}^{+} \mathrm{T}$ cells indicating that this transporter regulates the immune response.

In the following talk, Dr Rebecca Mather, a postdoctoral research associate at The Open University, then spoke about the long noncoding RNA landscape of NEPC $[9,10]$. This was approached from a screen of long noncoding RNAs in PDX models and clinical samples using databases prioritizing dysregulated transcripts which were evolutionary conserved and upregulated in NEPC. One of these transcripts NEARI was functionally characterized using genetic techniques and was shown to regulate cell proliferation, survival and invasion. Mechanistically, NEAR1 was shown to act by driving transcription of the pioneer transcription factor and proposed NEPC biomarker, FOXA2. Next-generation anti-sense oligonucleotides are also being developed against this transcript as a possible therapy and this transcript also shows promise as a urinary biomarker.

Finally, Dr Elena Jachetti, researcher at The Istituto Nazionale dei Tumori (Milan, Italy) gave a lecture on stromal accomplices driving neuroendocrine features in prostate cancer. Using the TRAMP mouse models of prostate cancer, Jachetti focused on the mast cell component of the tumors, showing that well-differentiated prostate adenocarcinomas need mast cells for in vivo tumor growth by provision of the matrix metalloprotease MMP9. Jachetti then blocked mast cell degranulation (and therefore MMP9) in these models and found that despite the effect on adenocarcinoma growth, NEPC arose in these mice with greater incidence. Osteopontin, was also discussed as another mediator released by mast cells. Indeed, Jachetti showed that TRAMP mice knock-outs of osteopontin also had increased NEPC incidence. When Jachetti then injected mast cells into these mice, the mast cells which were wild-type for osteopontin, but not osteopontin knock-out, reduced the frequency of NEPC tumors, indicating that osteopontin from mast cell was essential for this process [11,12]. 


\section{Conclusion}

In conclusion, the symposium has been a unique occasion to gather scientists and clinicians working on treatmentemergent NEPC and to propose new molecularly driven deadlines to accelerate research and improve the treatment of this deadly and poorly recognized malignancy. Key findings from this symposium are the new guidelines for the prompt diagnosis and management of treatment-emergent NEPC, as well as molecular evidence indicating the importance of epigenetic factors, noncoding RNAs and tumor niche in the development of this deadly disease.

\section{Financial \& competing interests disclosure}

FC has receieved a Cancer Research UK grant on Neuroendocrine prostate Cancer (Drug Discovery). The authors have no relevant affiliations or financial involvement with any organization or entity with a financial interest in or financial conflict with the subject matter or materials discussed in the manuscript.

No writing assistance was utilized in the production of this manuscript.

\section{References}

1. Aggarwal R, Huang J, Alumkal JJ et al. Clinical and genomic characterization of treatment-emergent small-cell neuroendocrine prostate cancer: a multi-institutional prospective study. J. Clin. Oncol. 36(24), 2492-2503 (2018).

2. Crea F. Neuroendocrine prostate cancer: long noncoding RNAs to treat an incurable cancer - an interview with Dr Francesco Crea. Epigenomics 11(13), 1461-1462 (2019).

3. Crea F, Venalainen E, Ci X et al. The role of epigenetics and long noncoding RNA MIAT in neuroendocrine prostate cancer. Epigenomics 8(5), 721-731 (2016).

4. Beltran H, Prandi D, Mosquera JM et al. Divergent clonal evolution of castration-resistant neuroendocrine prostate cancer. Nat. Med. 22(3), 298-305 (2016).

5. Lin D, Wyatt AW, Xue $\mathrm{H}$ et al. High fidelity patient-derived xenografts for accelerating prostate cancer discovery and drug development. Cancer Res. 74(4), 1272-1283 (2014).

6. Ci X, Hao J, Dong X et al. Heterochromatin protein $1 \alpha$ mediates development and aggressiveness of neuroendocrine prostate cancer. Cancer Res. 78(10), 2691-2704 (2018).

7. Choi SY, Xue H, Wu R et al. The MCT4 gene: a novel, potential target for therapy of advanced prostate cancer. Clin Cancer Res. 22(11), 2721-2733 (2016).

8. Choi SYC, Ettinger SL, Lin D et al. Targeting MCT4 to reduce lactic acid secretion and glycolysis for treatment of neuroendocrine prostate cancer. Cancer Med. 7(7), 3385-3392 (2018).

9. Crea F, Clermont PL, Parolia A, Wang Y, Helgason CD. The noncoding transcriptome as a dynamic regulator of cancer metastasis. Cancer Metastasis Rev. 33(1), 1-16 (2014).

10. Mather RL, Wang Y, Crea F. Is HOTAIR really involved in neuroendocrine prostate cancer differentiation? Epigenomics 10(10), 1259-1261 (2018).

11. Mauri G, Jachetti E, Comuzzi B et al. Genetic deletion of osteopontin in TRAMP mice skews prostate carcinogenesis from adenocarcinoma to aggressive human-like neuroendocrine cancers. Oncotarget 7(4), 3905-3920 (2016).

12. Pittoni P, Tripodo C, Piconese S et al. Mast cell targeting hampers prostate adenocarcinoma development but promotes the occurrence of highly malignant neuroendocrine cancers. Cancer Res. 71(18), 5987-5997 (2011). 
